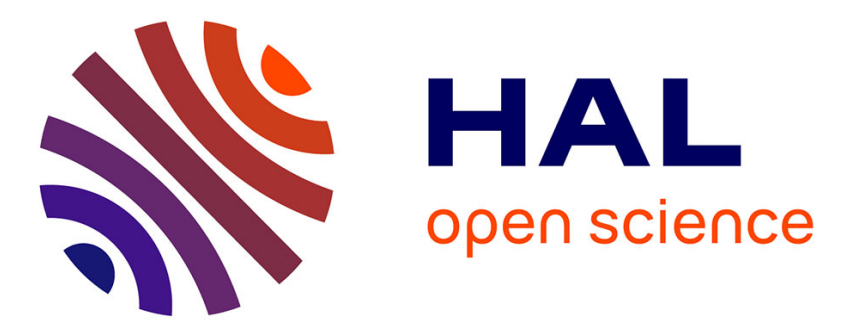

\title{
Atomistic response of a model silica glass under shear and pressure
}

B. Mantisi, Arnaud Tanguy, G. Kermouche, E. Barthel

\section{To cite this version:}

B. Mantisi, Arnaud Tanguy, G. Kermouche, E. Barthel. Atomistic response of a model silica glass under shear and pressure. The European Physical Journal B: Condensed Matter and Complex Systems, 2012, 85 (9), pp.85:304. 10.1140/epjb/e2012-30317-6 . hal-00731348

\section{HAL Id: hal-00731348 \\ https://hal.science/hal-00731348}

Submitted on 12 Sep 2012

HAL is a multi-disciplinary open access archive for the deposit and dissemination of scientific research documents, whether they are published or not. The documents may come from teaching and research institutions in France or abroad, or from public or private research centers.
L'archive ouverte pluridisciplinaire HAL, est destinée au dépôt et à la diffusion de documents scientifiques de niveau recherche, publiés ou non, émanant des établissements d'enseignement et de recherche français ou étrangers, des laboratoires publics ou privés. 


\title{
Atomistic response of a model silica glass under shear and pressure
}

\author{
B. Mantisi and A. Tanguy \\ Laboratoire de Physique de la Matière Condensée et Nanostructures, Université Lyon 1; \\ CNRS; UMR 5586 Domaine Scientifique de la Doua, F-69622 Villeurbanne Cedex, France \\ G. Kermouche \\ Ecole Nationale d'Ingénieurs de Saint-Etienne, Laboratoire de Tribologie et Dynamique des Systèmes, \\ UMR 5313 CNRS/ECL/ENISE, 58 rue J. Parot 42023 Saint-Etienne Cedex 2, France \\ E. Barthel \\ Surface du Verre et Interfaces, CNRS/Saint-Gobain, UMR 125, \\ 39 Quai Lucien Lefranc, 93303 Aubervilliers cedex, France
}

\begin{abstract}
The Mechanical Response of a Model Silica Glass is studied extensively at the submicrometer scale, with the help of atomistic simulations. The analysis of the response to a hydrostatic compression is compared to recent experimental results. The irreversible behaviour and the variation of intertetrahedral angles is recovered. It is shown that the atomistic response is homogeneous upon compression, in opposition with the localization along shear bands occuring during shear deformation with constant volume. Moreover, the Bulk Modulus anomaly is interpreted as due to a succession of such homogeneous but irreversible atomic rearrangements.
\end{abstract}

\section{INTRODUCTION}

Glasses feature remarkable mechanical responses which challenge modeling, among which we note the very high elastic limit $[29,43]$ and the localization of plastic deformation [35, 68, 74]. Beyond the static mechanical response, let us also mention the anomalous density of low frequency vibrational states giving rise to high heat capacity [24], low heat conductivity [24], low vibrational mean free path for the high frequency vibrational modes [28, 61, 69].

There are at least two reasons why the mechanical behaviour of glasses is more difficult to model than the mechanical behaviour of crystalline materials. One is the difficulty to identify a typical defect responsible for plastic deformation $[1,3,4,65]$; another is the lack of salient characteristic lengthscale in the disordered structure $[21,22,40,72]$.

In the past decades, much progress has taken place, and numerous theoretical studies of the mechanical behaviour of glasses at small scale $[6,19,35,39,60,64,67]$ have underlined common features shared by different kinds of amorphous materials. It is now well known that the elastic and acoustic response of glasses is strongly related to the inhomogeneous strain field taking place in these disordered materials, with nanometer-size correlational domains $[5,16,37,61,62,69]$. The plastic response is very different from the elastic one, even if prepared by the inhomogeneous elastic strain field [69]. The actual description of the plastic response of amorphous materials converges into a description in terms of local Shear Transformations [2] (ST) involving only few atoms, whose spatio-temporal dynamics can give rise to large scale deformations, for example along shear bands [35, 68].

However, the detailed study of the spatio-temporal dynamics of the Shear Transformations shows that the re- lation between local dynamics and local structure is not so evident. As an example, it has been shown through atomistic simulations in the athermal regime, that the relation between the existence of a local coordination defect and the occurrence of a Shear Transformation is dependent on the directionality of interatomic interactions $[20,66]$. In fact, nanoindentation experiments reveal also different behaviours depending on the composition of the glass. Silicate glasses for example, like soda lime silica glass, display shear bands when submitted to micro-indentation above the elastic limit, while pure silica glasses follow an anomalous behaviour with local densification and the formation of a Hertzian cone around the indent that is enhanced under shear $[15,23,30,54,55]$. This means that, when submitted to complex mechanical deformations combining local shear and pressure, the behaviour of a glass can be qualitatively very different for different compositions. Note also that the sensitivity of the detailed acoustic response to pressure is not easy to understand, since the simple scaling by the pressure dependence of the sound wave velocities is not always sufficient to understand the frequency shift of the vibrational density of states upon pressure $[45,49,50]$. It is thus very important to differentiate the roles played respectively by compression and shear at a atomistic level [71].

In this paper, we study the mechanical response of a model glass by numerical atomistic simulations. We use relatively realistic potentials and show that our results qualitatively emulate the main features of silica glass both in the elastic and the plastic regimes, under shear or hydrostatic pressure. 


\section{THE MODEL GLASS}

In order to understand how the atomic rearrangements affect the macroscopic behaviour of silica glasses, we have performed Molecular Dynamics simulations for different bulk sizes. Our systems are cubic boxes that contain 3000, 24000, 81000, 192000 and even 375000 atoms and respectively measuring $3.583,7.166,10.749$, 14.332, $17.950 \mathrm{~nm}$ edge size. These very large sizes are indeed necessary to avoid finite size effects due to long-range collective motion taking place in amorphous systems especially when submitted to low velocity mechanical deformation $[67,73]$. The key point of these simulation techniques is the empirical potential that governs the classical description of the interactions between atoms. The literature gives a large way to model silicates. It appears a general scheme, which is in fact the sum of different kinds of interactions. First of all there is the Coulombic part, describing electrostatic interactions between partial charges (ionic character of the bond) with $1 / \mathrm{r}$ long-range spatial dependence. A set of tools have been developed to deal with this long-range dependence. The most frequently used is the Ewald summation [18] for periodic boundary conditions, which consists in replacing the interaction in the real space by a rapidly convergent summation in the Fourier space. However, this can cost a lot of computation time. That is why some screened potentials have been used, like Yukawa potential for example [82], to represent the interaction between atoms at large distances. The Wolf truncation method [79] is another way to take into account long range interactions. It considers that for a certain distance, all the charges will be compensating each others. The second part in the potentials can take different forms, such as Morse [46], Buckingham [11] or Born-Huggins-Mayer [10]. It usually describes two kinds of interactions : repulsion at short range (which comes from Pauli repulsion between electronic clouds) and dispersion (which comes from the correlation between atoms charges fluctuations). This second term contains important parameters of the interaction, as the resulting potential wells characteristics (depth and width) : these characteristic parameters are fitted from ab-initio calculations [12]. Finally, because the second part is not enough to describe entirely the directionality of the bonds, one can add a three-body interaction part. It allows the atoms to be distributed around a mean angle value. The Sillinger-Weber three-body interaction is surely the most used, but one can construct a specific one, like Huang and Kieffer for example [26]. Although we had the choice among a large set of potentials, we had some constraints to take into account : 1) we wanted to study large samples and finite size effects; 2) for tractability, we also needed a potential with a simple analytical expression, and a range of interaction as short as possible. This led us to use a BKS potential [9] modified by A. Carré et al. [12], which has a Coulombic part plus a Buckingham repulsive potential, Wolf truncated, and smoothed. It can be described as :

$$
\begin{gathered}
\Phi_{\alpha \beta}^{B K S W}(r)=\Phi_{\alpha \beta}^{C o u l}(r)+\Phi_{\alpha \beta}^{B u c k}(r) \\
\Phi_{\alpha \beta}^{C o u l}(r)=q_{\alpha} q_{\beta} e^{2} V_{W}(r) G_{W}(r) \\
\Phi_{\alpha \beta}^{B u c k}(r)=\left\{A_{\alpha \beta} e^{-\frac{r}{\rho_{\alpha \beta}}}-\frac{C_{\alpha \beta}}{r^{6}}-\left[A_{\alpha \beta} e^{-\frac{r_{c, s h}}{\rho_{\alpha \beta}}}-\frac{C_{\alpha \beta}}{r_{c, s h}^{6}}\right]\right\} G_{s h}(r)
\end{gathered}
$$

with :

$$
\begin{gathered}
V_{W}(r)=\left(\left(\frac{1}{r}-\frac{1}{r_{c, W}}\right)+\frac{1}{r_{c, W}^{2}}\left(r-r_{c, W}\right)\right) \\
G_{W}(r)=\exp \left(-\frac{\gamma_{W}^{2}}{\left(r-r_{c, W}\right)^{2}}\right) \\
G_{s h}(r)=\exp \left(-\frac{\gamma_{s h}^{2}}{\left(r-r_{c, s h}\right)^{2}}\right)
\end{gathered}
$$

$\gamma_{s h}=\gamma_{W}=0.5, r_{c, W}=10.17 \AA$, and $r_{c, s h}=5.5 \AA$.

We also add a strong and regular repulsive part at short range $\left(r<r_{c, s r}\right)$ to avoid the collapse of atoms at high pressure, or high temperatures. The added repulsive part has the following form :

$$
\Phi_{\alpha \beta}^{R e p}(r)=\left\{\frac{D_{\alpha \beta}}{r}\right\}^{12}+E_{\alpha \beta} r+F_{\alpha \beta}
$$

The parameters of this potential are tabulated in table I.

\begin{tabular}{|l|c|c|c|c|}
\hline & $A_{\alpha \beta}(\mathrm{eV})$ & $\rho_{\alpha \beta}(\AA)$ & $C_{\alpha \beta}\left(e V . \AA^{6}\right)$ & $D_{\alpha \beta}\left(\AA . e V^{-12}\right)$ \\
\hline $\mathrm{O}-\mathrm{O}$ & 1388.773 & 0.3623 & 175.0 & 142.383338 \\
\hline $\mathrm{Si}-\mathrm{O}$ & 18003.7572 & 0.2052 & 133.5381 & 1.42402882 \\
\hline $\mathrm{Si}-\mathrm{Si}$ & 872360308.1 & 0.0657 & 23.299907 & 0.0 \\
\hline & $E_{\alpha \beta}\left(\mathrm{eV} . \AA^{-1}\right)$ & $F_{\alpha \beta}(\mathrm{eV})$ & $r_{c, s r}(\AA)$ & \\
\hline $\mathrm{O}-\mathrm{O}$ & -14.97811134 & 39.0602602165 & 1.75 & \\
\hline $\mathrm{Si}-\mathrm{O}$ & -3.24749265 & -15.86902056 & 1.27 & \\
\hline Si-Si & 0.0 & 0.0 & 0.0 & \\
\hline
\end{tabular}

TABLE I: Parameters of the empirical potential used to model the silica glass.

$D_{\alpha \beta}, E_{\alpha \beta}$ and $F_{\alpha \beta}$ have been adjusted in order to have the first, and second derivatives of the potential continuous.

The sample preparation proceeds as follows. The glasses are obtained from a crystal state which is heated and then quenched. We start from a $\beta$-Cristobalite 
crystal sample, which is the last crystalline state before the melting point at normal pressure. Then we heat the sample up to $5200 \mathrm{~K}$ during $1 \mathrm{~ns}$, let it evolve at constant temperature during 10000 time steps (time step $\delta t=10^{-15} \mathrm{~s}$ ), and quench it at $0 \mathrm{~K}$ in $1 \mathrm{~ns}$. The quenching rate is hence $5.2 .10^{12} \mathrm{~K} . \mathrm{s}^{-1}$. Finally we relax the simulation box to avoid residual stress, and then obtain a density of $2.18 \mathrm{~g} . \mathrm{cm}^{-2}$ with a pressure $P \approx 0 G P a$ with a precision of $10 M P a$.

In order to validate our sample, we check its structural properties. The pair correlation function (Fig. 1a) is in good agreement with former numerical [37, 70, 77] and experimental studies $[32,47,80]$ with a first $\mathrm{Si}-\mathrm{O}$ distance at $1.6 \AA$, a first $\mathrm{O}-\mathrm{O}$ distance at $2.6 \AA$ and a first $\mathrm{Si}-\mathrm{Si}$ distance at $3.2 \AA$. Regarding the angle distribution, the literature shows a distribution from $142^{\circ}$ to $153^{\circ}$ for the Si-O-Si angles from numerical $[8,26,37,53,70,76,77]$ as well as from experimental data [13, 14, 41, 47, 48, 56, 57]. This angle is the inter-tetrahedral angle. It can be seen as the link between all the elementary pieces in silica, as such it should be temperature and pressure dependent. Nevertheless, the angle distribution we found (Fig. 1b and Tab. II) contains the experimental and theoretical range of angles.

In this study, we have chosen to perform the mechanical deformation in the a-thermal regime, i.e. we either perform energy minimization $(T=0 \mathrm{~K})$, or we let our glass evolve at a temperature $T=10^{-5} \mathrm{~K}$, temperature at which thermal effects are totally negligible and not high enough to activate plastic events [73]. The reason is first that the choice of a numerical thermostat in mechanically driven systems is not a simple issue [59], and second because the object of this study is to show evidence of the effect of pure structural disorder and of the external load (shear vs. hydrostatic pressure) on the small scale deformation of the sample.

We then apply two kinds of mechanical loads : hydrostatic compression and shear at constant volume (shear at constant pressure will be the subject of another study). For the hydrostatic compression we applied an external pressure by two different ways. First, we apply successive pressure steps $P \rightarrow P+\Delta P$. At each pressure, we let the system evolve in the NPT ensemble with $T=10^{-5} \mathrm{~K} \mathrm{du}-$ ring a time $\Delta t=2.10^{-11} s$ (20000 time steps) that is sufficient to equilibrate the pressure through a Berendsen Thermostat. The pressure step used is $\Delta P=100 \mathrm{Bar}$ for pressures ranging from 0 to $12 G P a$ and then $\Delta P=$ 500 Bar up to $30 G P a$. In this case, the pressure increases monotonously during compression, and the deformation is applied dynamically with a given pressure variation rate $\Delta \dot{P} \approx 5-25.10^{12}$ Bar. $^{-1}$. The second way we have used to perform a hydrostatic compression, is to find the nearest local minimum of the total energy of the system. This kind of applied deformation corresponds to a quasistatic deformation at controlled volume. In order to circumvent the pressure variations, we first perform energy minimization at a desired external pressure. The minimization technique proposed by LAMMPS [33] consists in minimizing the energy $E_{t o t}=U+P\left(V-V_{o}\right)$, where $U$ is the internal energy of the system alone, $P$ is the desired pressure, and $V$ the volume to be reached. According to Parinello and Rahman [51], the system is coupled to an external variable that acts as a piston. We have compared different pressure increments : $P_{I n c}=5,25$ and 500Bar. It is seen that sometimes this pressure increment is not sufficient to overcome the energy barriers, and it is then increased by amounts of $P_{\text {Inc }}$ up to reaching a new equilibrium. Then we check the complete equilibrium of the system at constant volume. For that, the piston is removed, and the total energy is minimized at constant volume. The total force on each atom is then 0 , within the numerical accuracy. For both methods, we perform an increase until a given pressure $\left(P_{\max }\right)$, and then decrease until a zero pressure. This is done for a set of pressures $P_{\text {max }}(2,5,7,9,11,13,15,17,19,21,23$ and $25 \mathrm{GPa})$. We also focus on the history/memory effects by cycling loads, i.e. we start from $0 \mathrm{GPa}$ until $5 \mathrm{GPa}$, then decrease to 0 $\mathrm{GPa}$, increase from that state to $7 \mathrm{GPa}$, decrease to 0 GPa, and so on, for the same set of pressures $P_{\max }$. This allows to compare the behaviour for increasing and decreasing pressures and test the sensitivity of the Bulk Modulus and of the yield stress to the loading history. For the shear at constant volume, we change the shape of the box within a monoclinic symetry and a small deformation step, and then let the atoms relax by minimizing the total energy in the box. We carry on this operation to get the desired total shear deformation. The shear step corresponds to a deformation of $\Delta \epsilon_{x y}=10^{-4}$, that is small enough to decorrelate the plastic events that occur during the shear [36]. These two first loads are configurational minimizations. It corresponds to a very fast relaxation time between each strain step (quasi-static deformation) and mechanical equilibrium (athermal response). It gives very detailed results on the restructuration of the phase space upon mechanical deformation [59], and allows to construct the landscape over which thermal activation acts. Thermal agitation, that can play a role even below the glass transition temperature [59], will thus not be discussed here.

\begin{tabular}{|c|c|c|c|c|}
\hline & $0 \mathrm{GPa}$ & $5 \mathrm{GPa}$ & $10 \mathrm{GPa}$ & $15 \mathrm{GPa}$ \\
\hline Si-O-Si & 151.5 & 145.68 & 137.44 & 133.93 \\
& $144[47] 148.3[48]$ & $137.40[17]$ & $135.01[17]$ & - \\
& $152[13]$ & & & \\
\hline O-Si-O & 109.5 & 108.36 & 108.03 & 106.9 \\
& $109.5[47] 109.47[48]$ & - & - & - \\
& $109.7[13]$ & & & \\
\hline
\end{tabular}

TABLE II: Values in degres of the maxima in the distribution of $\mathrm{Si}-\mathrm{O}-\mathrm{Si}$ and O-Si-O angles. Data from the present simulations are compared to the data given in the literature. 

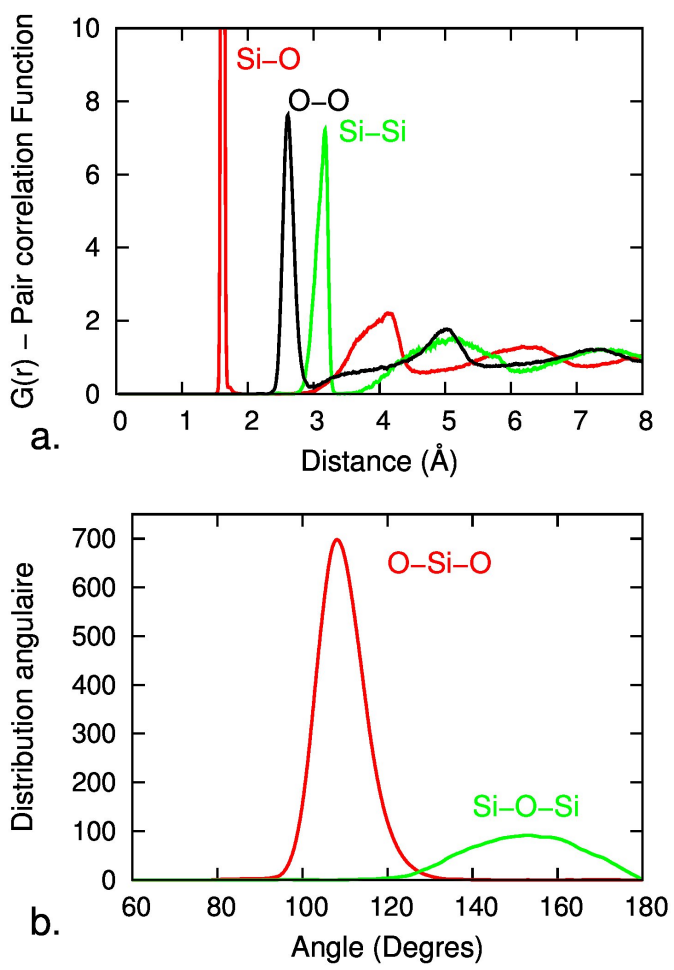

Figure 1: [Color Online] a) Pair Correlation Function for the species $\mathrm{O}-\mathrm{O}, \mathrm{Si}-\mathrm{O}$ and $\mathrm{Si}-\mathrm{Si}$ in a sample with $\mathrm{P}=0 \mathrm{GPa} . \mathrm{b}$ ) Angles distribution $\mathrm{Si}-\mathrm{O}-\mathrm{Si}$ and $\mathrm{O}-\mathrm{Si}-\mathrm{O}$ in a sample with $\mathrm{P}=0$ GPa.

\section{HYDROSTATIC COMPRESSION}

The hydrostatic compression has been applied following the two methods detailed in the previous part (equilibrium at very small temperature with a Berendsen Barostat and Thermostat, or energy minimization at controlled volume). We have found that both methods give the same general variation $P(\Delta V / V)$, thus confirming that the temperature $\left(T=10^{-5} K\right)$ chosen for the first method corresponds to athermal simulations. However, the dynamical method (Berendsen thermostat) shows a monotonous increase of pressure, whereas the quasi-static volume controlled protocole shows pressure jumps due to local irreversible rearrangements (Fig. 2a). The irreversibility of these rearrangements has been checked by comparing the atomic positions in the configuration just before the jump, and the atomic positions in the configuration obtained by decreasing the volume again after the jump up to its initial value (Fig. 4-c). It shows irreversible local quadrupolar rearrangements corresponding to irreversible micro-shears that do not affect the global volume, but storing a small amount of dissipated energy. The number of these rearrangements within a pressure window $\Delta P=0.1 G P a$ is not dependent on the increment $P_{\text {Inc }} \leq 25$ bar (Fig. 3-c). It represents the number of dissipative rearrangements needed to over-
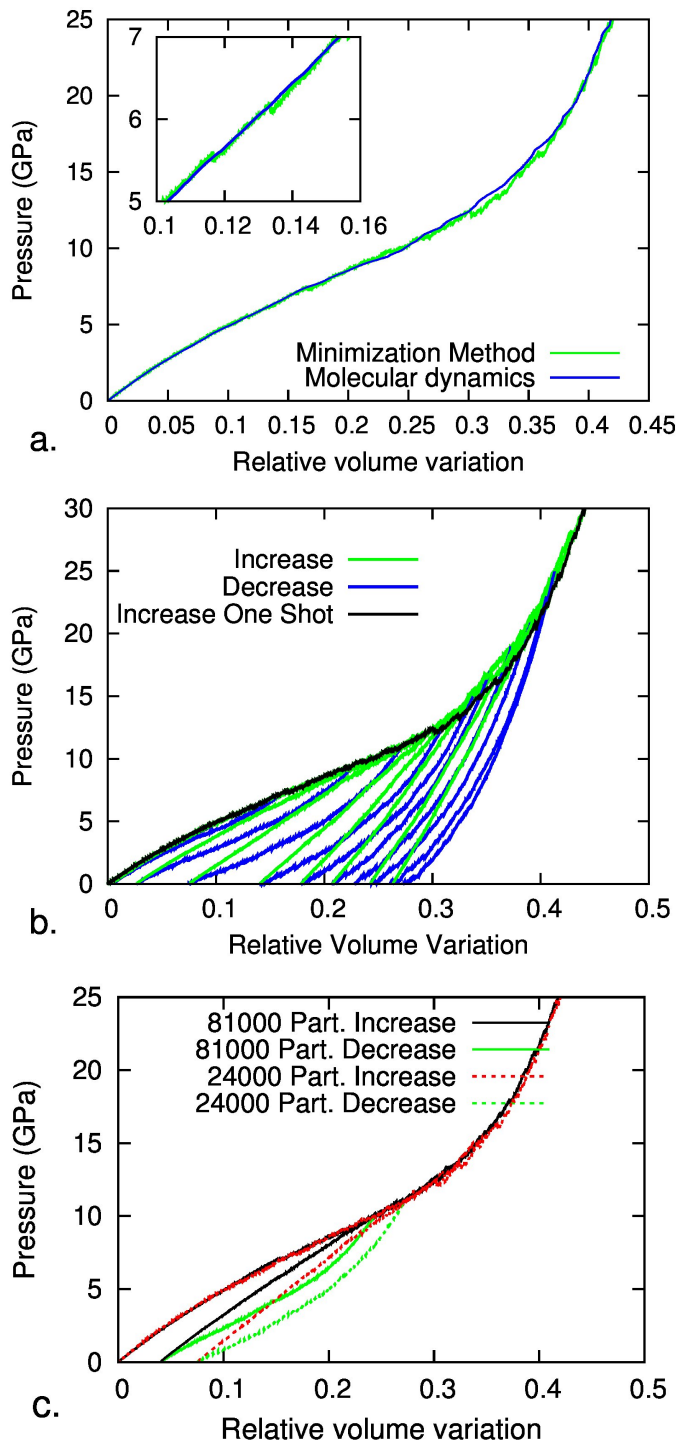

Figure 2: [Color Online] a) Pressure as a function of the relative Volume variation. Comparison between the Dynamical protocole at controlled pressure, and the quasi-static protocole at controlled volume. $\mathrm{N}=24000$. Quasi-static deformation at controlled volume shows pressure jumps related to irreversible local deformation (micro-plasticity). b) Pressure as a function of the relative Volume variation for simple compression and cyclic loads, at controlled volume. $\mathrm{N}=24$ 000. c) Same curves for $\mathrm{N}=24000$ and $\mathrm{N}=81000$.

come energy barriers during the minimization process. The corresponding pressure jumps are of course invisible in the dynamical curve obtained with the Berendsen thermostat since only the final pressures and volumes are drawn there. Therefore, we will focus only on the volume imposed protocole that contains more informations about small-scale rearrangements.

Then we have performed two types of hydrostatic compression tests on our model bulk silica glass. The first test is a single compression run up to the maximum 

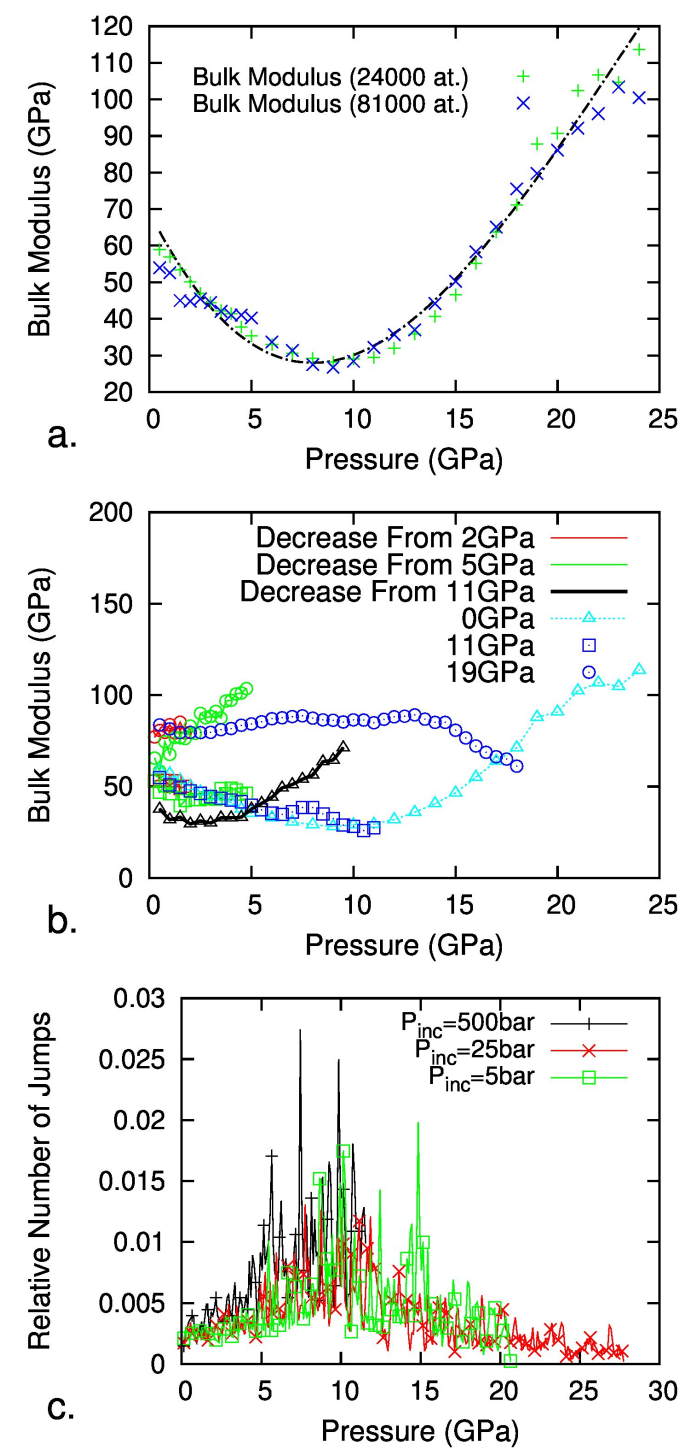

Figure 3: [Color Online] a) Bulk Modulus as a function of the pressure, from the data obtained in Fig. 2. The Bulk Modulus is defined as the local slope $-V \Delta P / \Delta V$ measured within a pressure interval $\delta P=0.1 G P a$, for $N=24000$ and $N=81000$ particles. Long dashed line : guide for the eyes. b) Comparison of the same Bulk Modulus obtained for a one shot deformation : for a simple increase from $P=0 G P a$ (triangles), for a configuration that has been densified previously up to $11 \mathrm{GPa}$ (squares), for a configuration that has been previously densified up to $19 \mathrm{GPa}$ (circles), and the corresponding decreases : from $2 G P a$ (red), $5 G P a$ (green) and $11 G P a$ (black). Note the marked irreversibility for the configuration previously densified up to $19 \mathrm{GPa}$, or for the drecrease from $11 G P a$. c) Number of jumps measured during pressure intervals of amplitude $\delta P=0.1 G P a$ as a function of the applied pressure, and divided by the total number of pressure steps imposed to the sample in the same interval.

pressure $P_{\max }=30 \mathrm{GPa}$. The second test is a series of partial compression-(loading-unloading) runs with in-
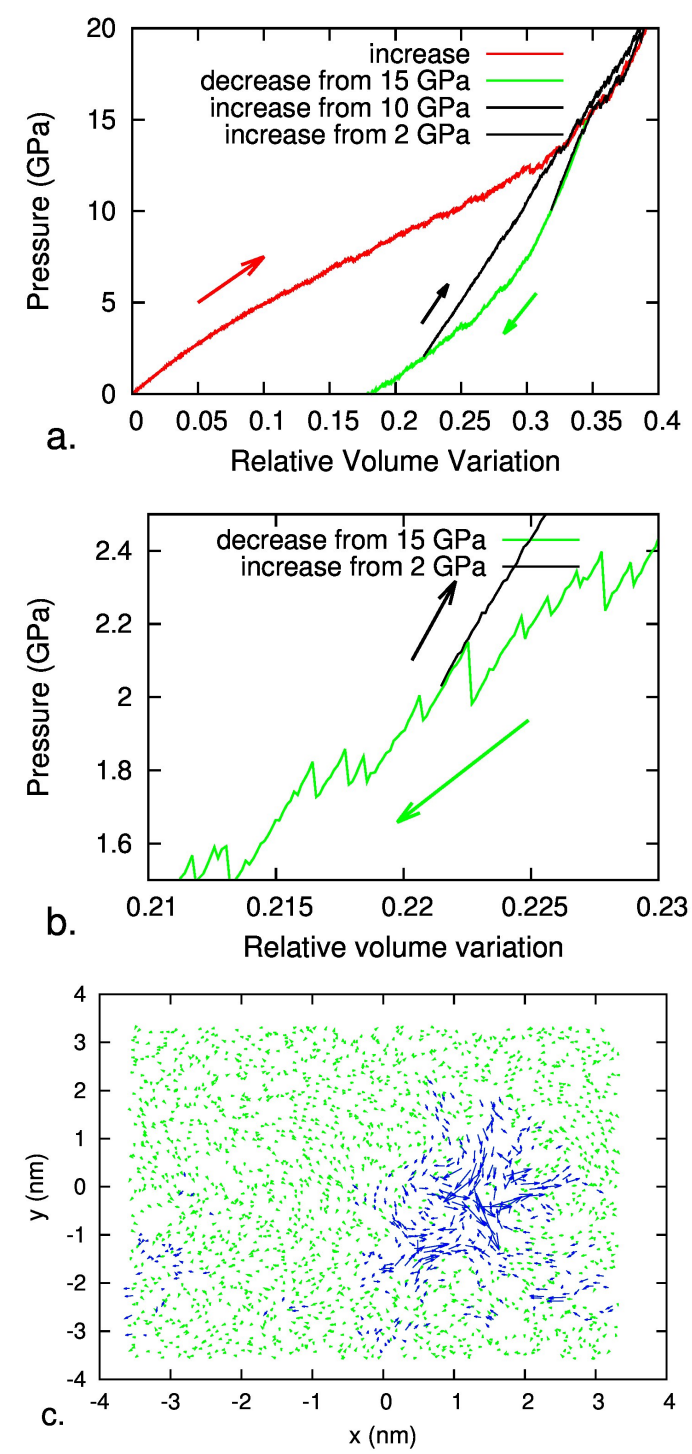

Figure 4: [Color Online] a) Pressure as a function of the relative volume variation during an increase from $0 G P a$, then a decrease from $11 G P a$ followed by an increase from $2 G P a$. The aperture of the cycle is clearly visible. b) Zoom of the same curve close to $2 \mathrm{GPa}$. Note that the pressure increase follows the local slopes. c) Residual displacement of all the atoms of the sample in projection in the $x-y$ plane, when the volume comes back to its initial value, and after a pressure jump. The amplitude of arrows is proportional to the atomic displacement, with a magnification of 10 in comparison with the scale used to represent the positions (at the origin of the arrows). Blue arrows represent the largest 3 percents displacement amplitude particles.

creasing maximum pressure. In Fig. 2-b, we plot the total average pressure with respect to the relative volume variation for these two tests. From this figure, we can see that for a single compression up to $30 \mathrm{GPa}$, the pressure exhibits globally a monotonous increase with the compressive strain (the volume decreases upon pressure), but 

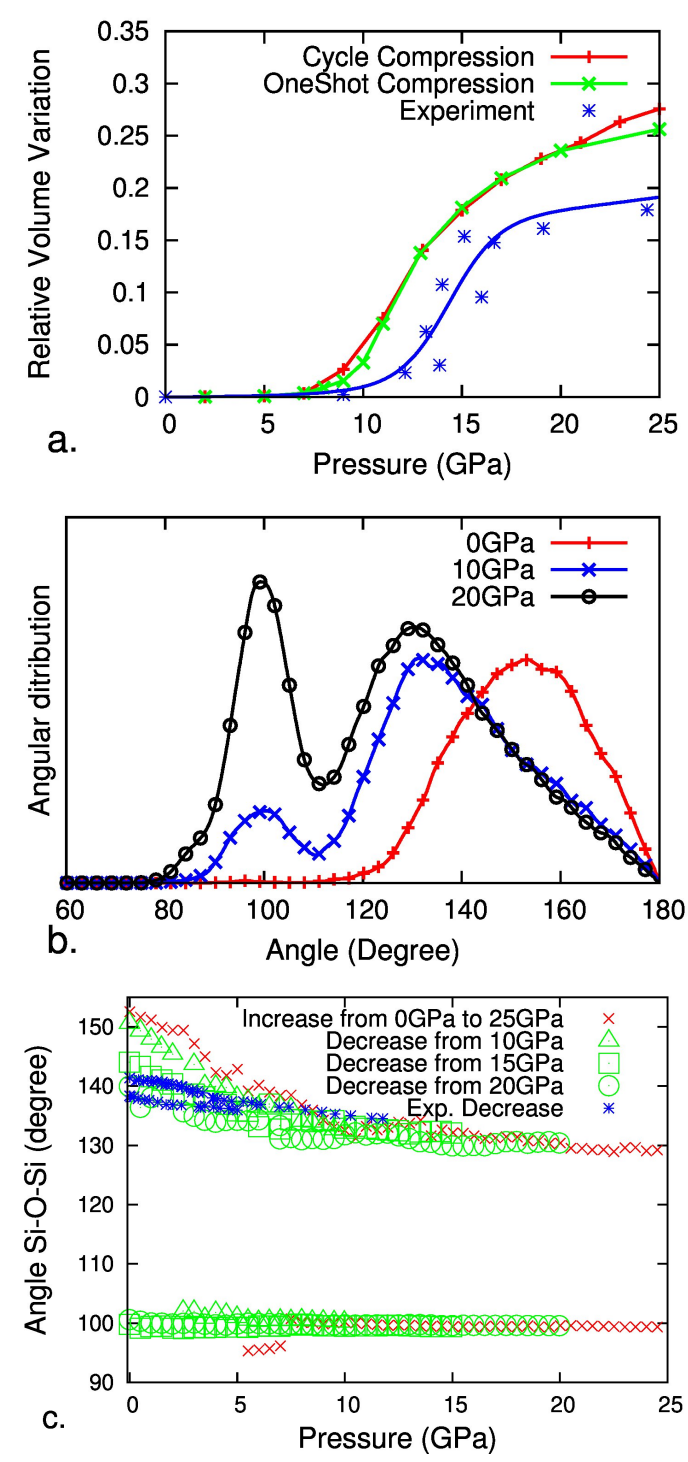

FIgURE 5: [Color Online] a) Residual Volume Variation as a function of $P_{\max }$ applied and comparison with experimental results obtained in [54]. b) Variation of the distribution of $\mathrm{Si}-\mathrm{O}-\mathrm{Si}$ angles during compression. c) Variation of the Si-O$\mathrm{Si}$ angles defined as the maxima in the angle distribution, and comparison with experimental results obtained in [17] for different $P_{\max }$.

with small fluctuations including small pressure decreases (with amplitude $\delta P \approx 0.01 G P a$ ) during the compression of the volume. As seen before, these small pressure decreases consist of plastic rearrangements. Note that a negative Bulk Modulus $(B \equiv-V . \Delta P / \Delta V)$ is not allowed by mechanical stability and corresponds necessarily to a mechanical instability. We have checked that these small pressure decreases are combined with a dissipative (plastic) micro-rearrangement, by looking at the irreversible components of the atomic displacements when the volume goes back to its initial value (Figure 4-c). We will comment again on this later. Let us come back first to
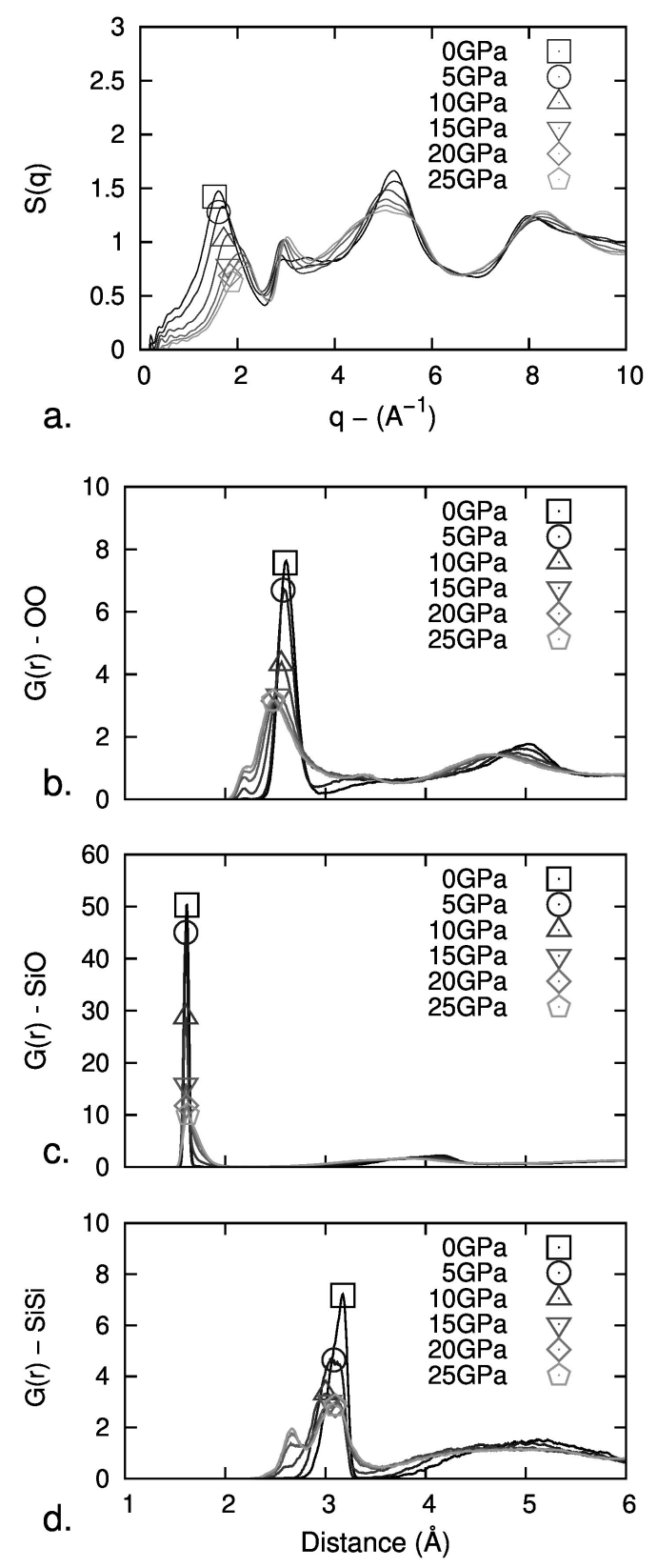

Figure 6: [Color Online] a) Angular average of the Static Structure Factor as a function of the applied pressure. b) Pair Distribution Functions of the species O-O, Si-O and $\mathrm{Si}-\mathrm{Si}$, as a function of the applied pressure.

the global $P-\Delta V / V$ curve shown in Fig. 2-b.

Considering the pressure fluctuations measured during the compression of our systems, we have first smoothed the curves with a resolution $\delta P=0.1 G P a$ in order to get an estimation of the macroscopic Bulk Modulus. The well known experimental measurement specific to silica is its strong anomalous elastic non-linearity : the Bulk Modulus is known to decrease first with the application of hydrostatic pressure up to about $2.5 \mathrm{GPa}$, then increases again $[17,31]$. This mechanical behaviour has no defi- 


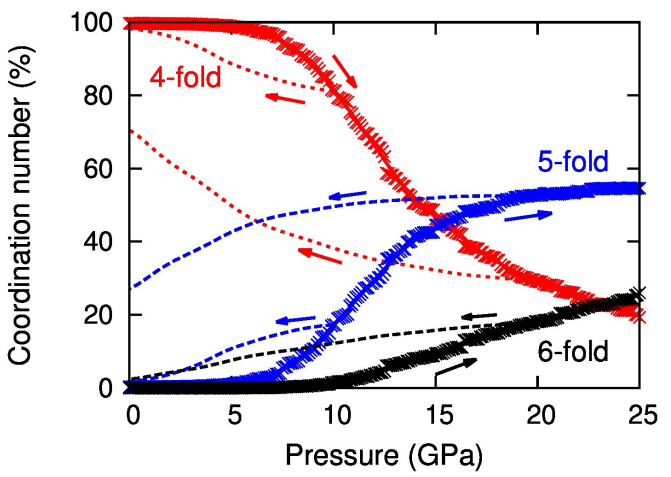

Figure 7: [Color Online] Coordination number of Si atoms as a function of the applied pressure, during an increase and two decreases from $9 G P a$ and from $19 G P a$.

nite structural explanation, even if some authors have proposed to relate it to a subtle morphological change in the shape of Si-rings and to a partial $\beta$ to $\alpha$ transition $[26,81]$. We have calculated the macroscopic Bulk Modulus from the slope of the single compression curve $P-\Delta V / V$ smoothed with a resolution $\delta P=0.1 G P a$. There is a complex variation of the slope of this smoothed $P-\Delta V / V$ curve (reported in Fig. 3 -a) : the slope initially decreases then it increases again above roughly 8 GPa, resulting in an inflexion point. This corresponds to the well kown "elastic anomaly" observed experimentally in pure silica glasses. This behaviour captures qualitatively the anomalous non linear elastic response of silica glass, although the actual values (modulus, position of the minimum) differ by a factor 2-3. This decrease in the macroscopic Bulk Modulus is actually directly related to the number of micro-jumps in the detailed pressure response at controlled volume. The relative number of jumps computed in pressure windows of width $\delta P=0.1 G P a$ and divided by the total number of steps in that window is shown in Fig. 3-c. It shows a maximum close to $P=8 G P a$, independent on the increment $P_{\text {Inc }}$, and this large number of micro-jumps could thus be responsible for the apparent decay of the Bulk Modulus. Thus the well known "elastic anomaly" of silica glasses appears to result from a micro-plastic behaviour in our simulations. The evolution of this apparent Bulk Modulus can even differ strongly, depending on the plastic state of the system, as already shown experimentally [58]. We observe (Fig. 3-b) that the modulus increases with density as evidenced by a modulus of about $100 \mathrm{GPa}$ after full densification has been reached above $P_{\max }=25 \mathrm{GPa}$. The modulus is then independent of hydrostatic pressure. This is qualitatively in agreement with the Brillouin measurement $[58,83]$, from which an increase of the bulk modulus by 1.8 can be calculated from a ca. $20 \%$ density increase. For macroscopically plastically densified samples for example (Fig. 3-b, with $P_{\max }=19 G P a$ ) the elastic anomaly can even disappear.

It must as well be noticed that during decompression,
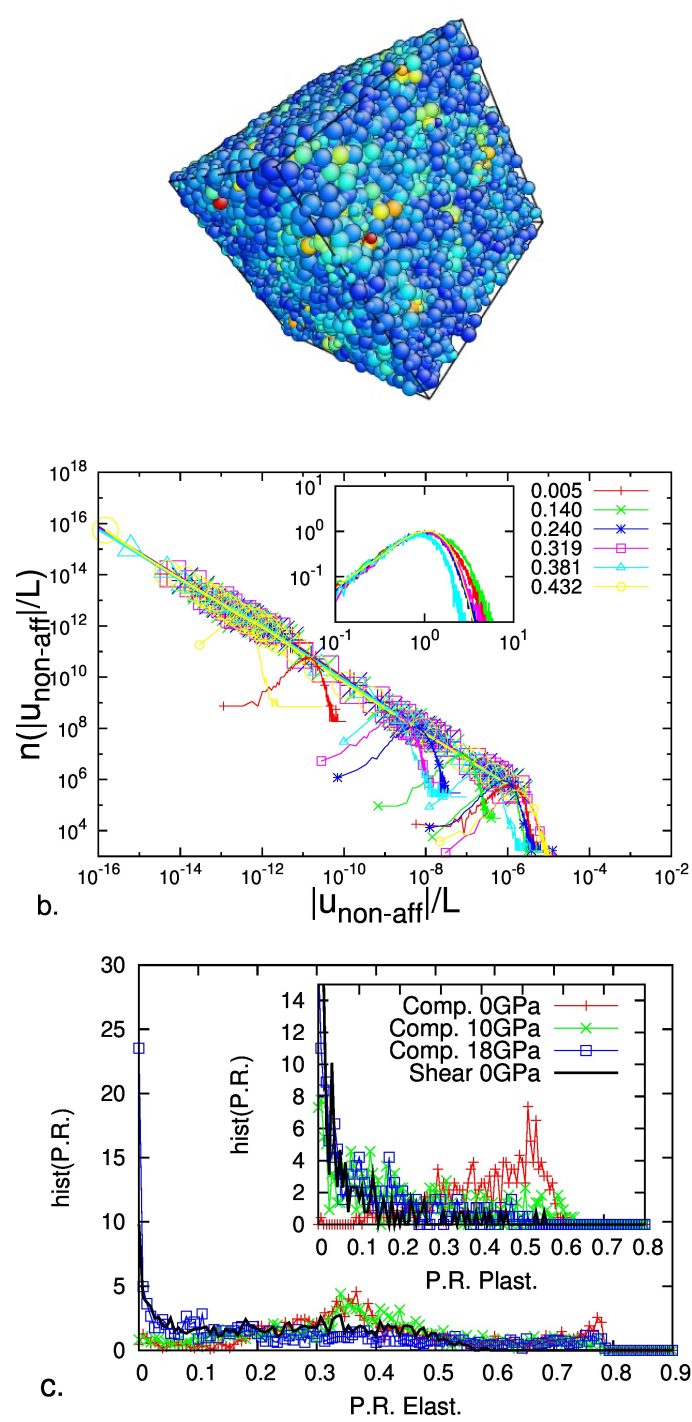

Figure 8: [Color Online] a) Amplitude of the Non-Affine displacement field (departure from homogeneous compression) in a typical irreversible step of the stress-strain curve during compression.(Picture realised with Atomeye [34]) b) Histogram of the amplitude of the Non-Affine displacement field measured on each atom for different steps of compression. The numbers in the legend correspond to the $\Delta V / V$ value. The points aligned are the maximum values of the histograms for different steps of pressure. Inset : Superposition of the curves with the help of a scaling factor. Note the similarity between the histograms for either reversible (elastic) and irreversible motion. c) Histogram of the participation ratio of the NonAffine displacement field during compression, compared to the same histogram upon shear deformation. The histogram is obtained on 2000 compression steps. The pressure mentioned indicate the average pressure $( \pm 0.5 G P a)$. Elastic rearrangements are in the main graph, and plastic rearrangements are in the inset.

this Bulk Modulus is very different from its value during 
compression (see figure 3-b). Here again, the different succession of instabilities during loading and unloading gives rise to an asymmetric behaviour with cycle aperture. For a decompression from $11 \mathrm{GPa}$ for example (triangles with bold line in Fig. 3-b), the minimum value of the Bulk Modulus is pushed back to 2.5 GPa, and even if the local Bulk Modulus is the same at the beginning of the decompression, the macroscopic plastic behaviour manifests itself by a jump in the macroscopic Bulk Modulus, if $P_{\max }$ is sufficiently high (see in Fig. 3-b, the curve corresponding to the decrease from $5 \mathrm{GPa}$ for the configuration obtained with $\left.P_{\max }=19 \mathrm{GPa}\right)$. This hysteretic behaviour is usually described in terms of internal friction [44]. We propose here a microscopic description of what happens during the hysteretic process.

The analysis of the Bulk Modulus can be completed by a detailed analysis of the local $P-\Delta V / V$ slope (Fig. 4). It is shown in Fig. 4-b, that the local slope (Local Bulk Modulus) can differ drastically from the slope of the smoothed curve (Macroscopic Bulk Modulus). The difference is so high that when decreasing the pressure, the system follows elastically its local slope and this behaviour gives rise to the aperture of a cycle in the global $P-\Delta V / V$ curve (Fig. 4-a). When the pressure increases again (for example from $P=2 G P a$ in Fig. 4-b), the system follows its local slope and opens a new cycle. Suprisingly however, the system finally converges to the initial one-shot curve, thus proving that probably it keeps somehow the memory of its initial state, that is more precisely of the maximum pressure $P_{\max }$ it has reached previously. The series of loading-unloading cycles with increasing maximum pressure thus demonstrate that the single compression curve $P-\Delta V / V$ indeed reveals a well defined response of the material : after each unloading segment, the reloading curve converges to the single compression curve as soon as the maximum pressure of the previous cycle is reached. This means that even a complex loading history does not affect the relation between $P_{\max }$ and the relative volume variation. A similar anelastic behaviour has also been obtained by S. Ispas [27] on a similar system.

From the cyclic loads, we can also characterize the macroscopic plastic behaviour of the samples. We can indeed identify a pressure threshold above which the unloading curve for each cycle significantly departs from the single compression curve (Fig. 2-b), giving rise finally to an irreversible volume variation after the system has come back to its initial pressure. Plotting the residual volumetric deformation after decompression (Fig. 5-a) as a function of the maximum pressure $P_{\max }$ applied, the resulting strain hardening curve reveals that significant densification $(\Delta V / V>5 \%)$ is first observed around the macroscopic plastic threshold $P_{\max } \approx 11 G P a$. The densification also tends to flatten out around $20 \mathrm{GPa}$, at a value close to $25 \%$. This irreversible macroscopic volume variation is a clear signature of macroscopic irreversible response, i.e. plasticity. We can thus identify an approximately reversible regime (with internal friction) for $P_{\max }<11 G P a$ and a macroscopically plastic regime for $P_{\max }>11 G P a$.
Above this macroscopic plastic threshold, the deviation of the unloading curve from the single compression curve translates into a permanent residual volumetric deformation (densification). This behaviour is qualitatively comparable to the experimental results (see Fig. 5-a) obtained by Raman spectroscopy $[15,54,55]$.

The densification of the system upon pressure is accompanied by structural changes. First consider the Static Structure Factor $S(q)$ (Fig. 6-a). The main change in $S(q)$ consists of decreasing the peaks especially at large distances (small wave-vectors), which is evidence of a more homogeneous sample with a mixture of different lengthscales, in agreement with [42]. The large scale variations of $S(q)$ saturate when $P>11 G P a$ that is for permanent densification. At small distances, the pair distribution functions (Fig. 6-b) show a general decrease of bond lengths, plus an additional peak at small distances for Si-Si and O-O bonds, appearing at $\mathrm{P}=11 \mathrm{GPa}$. These structural changes are accompanied by angular distorsion (Fig. 5-b,c). The angle evolution is reported in Fig. 5-b. The most probable Si-O-Si angle decreases from $151^{\circ}$ to $130^{\circ}$ as a function of the pressure, and a second maximum at $100^{\circ}$ in the Si-O-Si angle's distribution appears from $P=2.5 \mathrm{GPa}$ first reversibly, and then permanently above $11 \mathrm{GPa}$. This evolution corresponds to different Si-centered tetrahedra moving closer to each other in two different ways. Note that the experimental results [17] reported in the figure 5-c support very well this evolution of the distribution of angles. The structural changes can also be quantified by looking at the evolution of the average Si-coordination number as a function of the pressure applied (Fig. 7). The amount of 4-fold coordination numbers decreases, whereas 5 -fold increases simultaneously with the angle's variation, qualitatively consistent with [42]. Note that this evolution is not fully reversible, since it follows a different path during compression and decompression, as does the macroscopic Bulk Modulus.

Finally, the mechanical behaviour of amorphous materials has often been characterized by its inhomogeneous displacement field [35, 39, 67]. This was done for shear deformation, since shear is usually considered as responsible for strain localization and shear banding [59]. The inhomogeneous deformation can also be responsible for the low value of the Elastic Moduli, as shown by Reuss long time ago [78]. For this reason we have analysed the displacement field as a function of load, and especially how it departs from the homogeneous strain field of an isotropic and homogeneous material. As an example, the amplitude of such a displacement field is shown in figure 8-a. The corresponding histogram of the amplitude of the displacements is shown in figure 8-b. It is clear that the distributions of displacements for reversible as well as for irreversible paths are very similar. They differ only by a scaling factor and superimpose into a master curve (inset Fig. 8-b).

Moreover, the amplitude of the displacements appears to be homogeneously spread over the sample. The spatial 
distribution of this amplitude can be quantified by the participation ratio (P.R.) defined as

$$
P R=\frac{1}{N} \frac{\left(\sum_{i=1}^{N} u_{i}^{2}\right)^{2}}{\sum_{i=1}^{N} u_{i}^{4}}
$$

that goes from $1 / N$ for the isolated motion of a single atom to 1 for a block motion of the full system. It is a measure of the percentage of atoms that move together. As shown in Fig.8-c, the participation ratio of the non-affine displacement field, in case of compression, has a very large distribution independent on the pressure for $P>0 G P a$, up to a maximum value $P . R \cdot \max \approx 80 \%$. Its distribution is not very different for plastic and for elastic rearrangements : it is very large in both cases. It corresponds to a fairly homogeneous response without localization. The width of the distribution of the P.R. is probably related to different numbers of maxima in the displacement amplitude, since the distribution of displacements is always the same (Fig.8-b). The absence of localization is inferred from the absence of size dependence in the P.R. (not shown here). The homogeneous spreading of the atomic displacements over the sample leads to a well defined spatial average for the total stress released during a plastic deformation (amplitude of the pressure drops visible in Fig. 2-c and Fig. 4-b), that has no size dependence, as well. These results are very specific of a hydrostatic compression. They are very different from what happens upon shear [68]. The latter case will be discussed now in detail.

\section{SHEAR}

In order to compare the elasto-plastic response of the same system upon compression and upon shear, we apply now a quasi-static shear to the sample through energy minimization at constant volume, with a monoclinic change of the box orientation (as we have already done in the past on many other systems $[20,59,66,68,72])$. The corresponding mechanical deformation is a simple shear. In this case, the sample is not allowed to densify, but it is possible to infer its tendency to densify by measuring the resulting average pressure inside the sample : negative pressure (i.e. tension) builds up to oppose the tendency to densify.

The corresponding stress-strain behaviour is depicted in Fig. 9 for different system sizes. The stress-strain curves show different regimes : first a linear (mainly reversible) part from which the shear modulus can be calculated. We find $\mu=\sigma_{x y} /\left(2 . \epsilon_{x y}\right) \approx 35 G P a$, in good agreement with experimental values; second, a maximum followed by stress softening; then a plastic plateau consisting in a series of small scale linear branches and stress decays. Contrary to the hydrostatic compression, the finite size effects are evident here. Histograms of stress jumps (Fig. 9-b) show a $1 / L^{\alpha}$ (with $\alpha \approx 2.25$ )
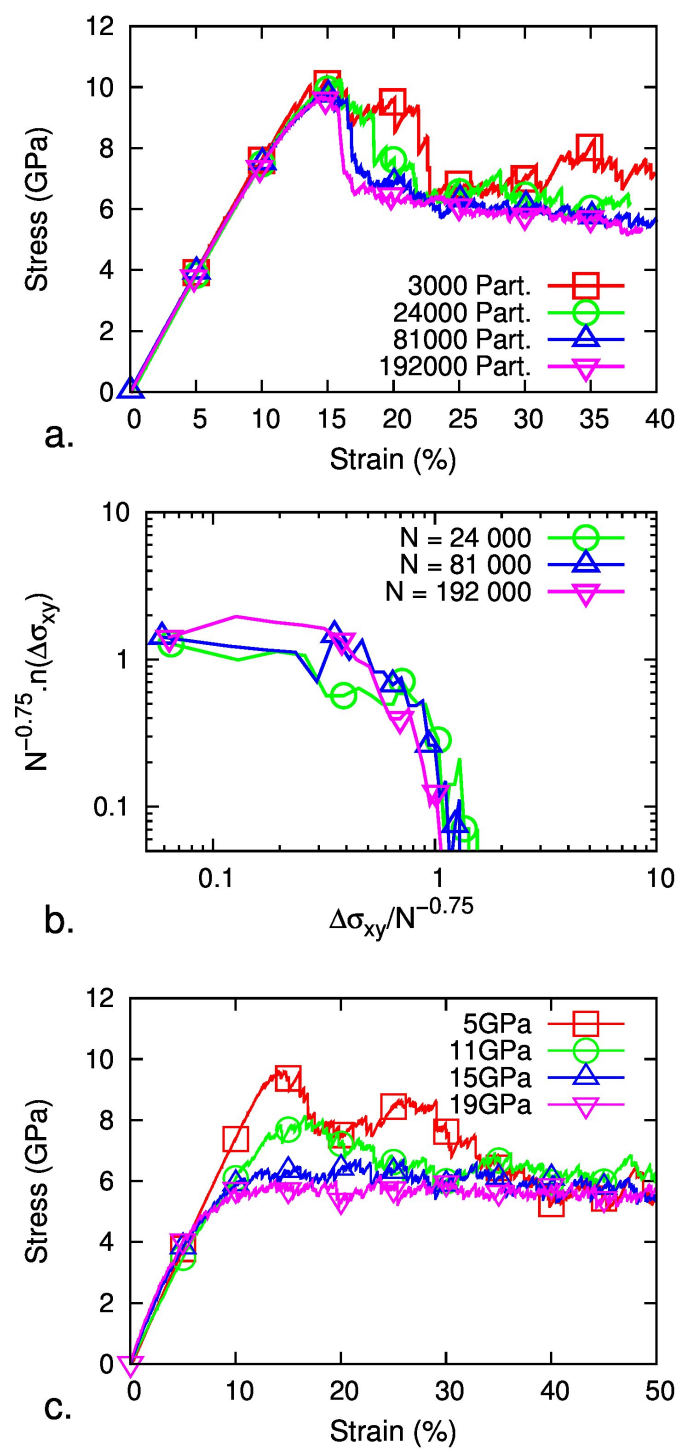

FIGURE 9: [Color Online] Shear stress $\sigma_{x y}$ as a function of the imposed shear strain $\epsilon_{x y}$ during a quasi-static simple shear at constant volume. a) for different system sizes $\mathrm{N}=3$ $000(\mathrm{~L}=3.583 \mathrm{~nm}), \mathrm{N}=24000(\mathrm{~L}=7.166 \mathrm{~nm}), \mathrm{N}=81000$ $(\mathrm{L}=10.748 \mathrm{~nm}), \mathrm{N}=192000(\mathrm{~L}=14.332 \mathrm{~nm})$ b) Histogram of Stress jumps in the plastic Plateau rescaled by $N^{0.75}$ (i.e. $L^{2.25}$ ). c) after different pressures have been applied to the sample $P_{\max }=0 G P a, P_{\max }=11 G P a, P_{\max }=$ $13 G P a, P_{\max }=15 G P a, P_{\max }=17 G P a, P_{\max }=19 G P a$ and $P_{\max }=21 G P a . N=24000$

dependence corresponding to defects neither localized in a small volume element $\left(\propto 1 / L^{3}\right)$ nor linear $\left(\propto L / L^{3}\right)$. This proves that dissipative rearrangements are localized, contrary to the case of hydrostatic compression where they were spanning the entire system and did not display any size dependence.

Stress-softening is usually related to permanent localization of dissipative rearrangements [59]. It is dependent on the initial configuration, which strongly depends on 

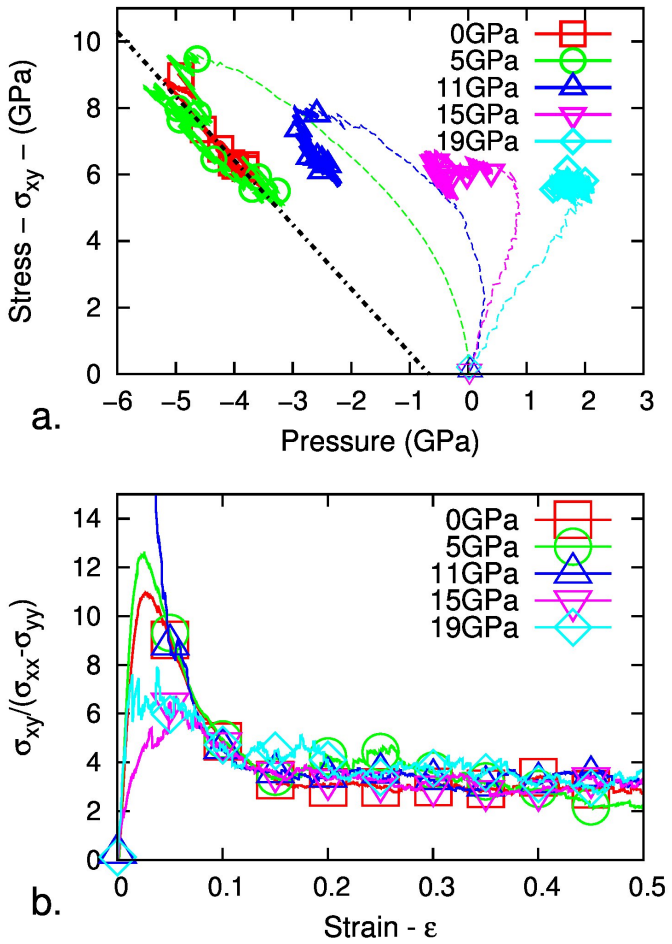

Figure 10: [Color Online] a) Shear stress as a function of the pressure during the shear transformation at constant volume, for samples densified at $P_{\max }=5,11,15$ and $19 \mathrm{GPa}$ respectively. Note the linear relation (Mohr-Coulomb like relation) with a negative coefficient after the Yield Stress has been reached. b) $\sigma_{x y} /\left(\sigma_{x x}-\sigma_{y y}\right)$ as a function of the shear strain for the same deformations.

sample preparation. Densification through application of a hydrostatic pressure larger than the macroscopic yield threshold will of course alter this initial configuration, and we want precisely to study its impact now. Fig. 9-c displays stress-strain curves in shear for several configurations that have been compressed up to different $P_{\max }$ and then relaxed to zero pressure before shear. We find that increasing the density in this way decreases the maximum shear stress. For $P_{\max }>17 G P a$ the stress overshoot even disappears completely. The explanation for that behaviour is that the configuration has then been sufficiently homogeneized to avoid permanent localization. Note that the disappearance of the stress overshoot is here connected to subtle variations in the sample structure, and not to a change of interatomic interactions [20] or deformation protocole [75]. This means that the shear softening shown here, depends on sample structure in a subtle way. In any case, we have observed here (see Fig. 12), that the absence of strain softening is related to the absence of permanent shear bands along the system. The more compressed is the initial sample, the more homogeneous is the corresponding plastic deformation upon shear.

For all the configurations considered, it is also in-
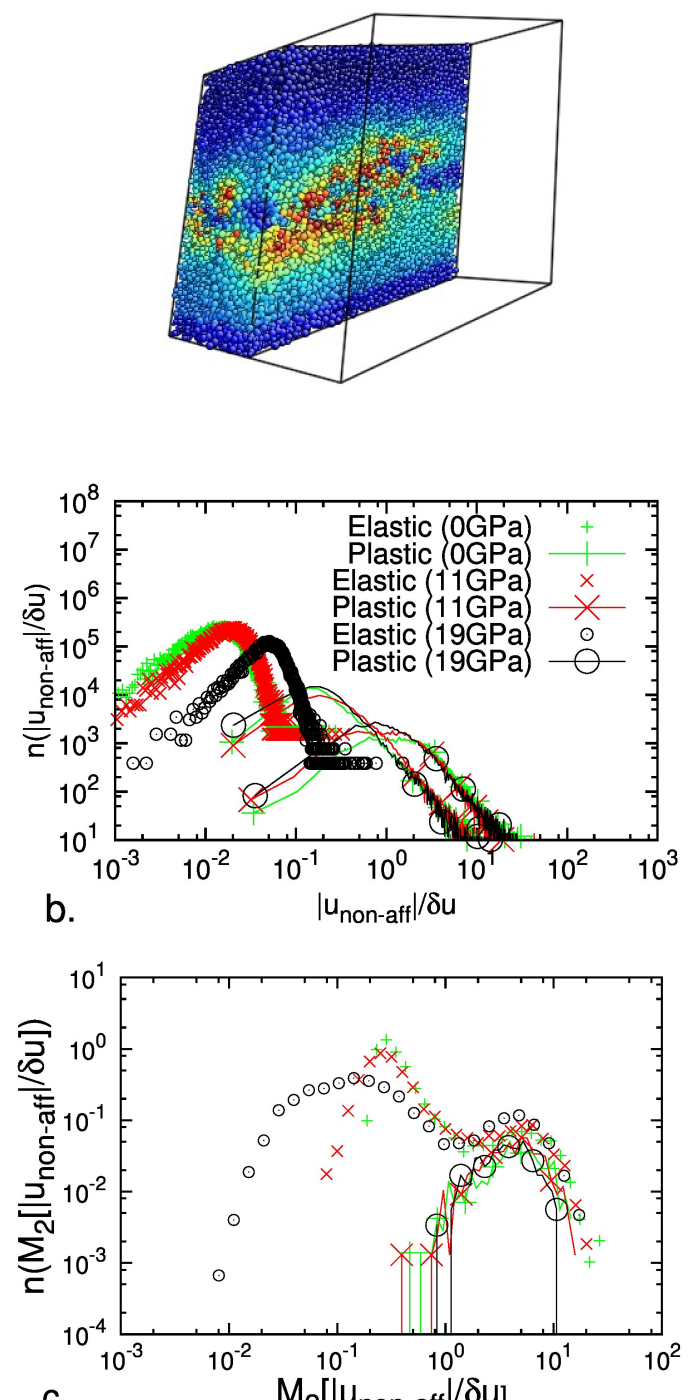

C.

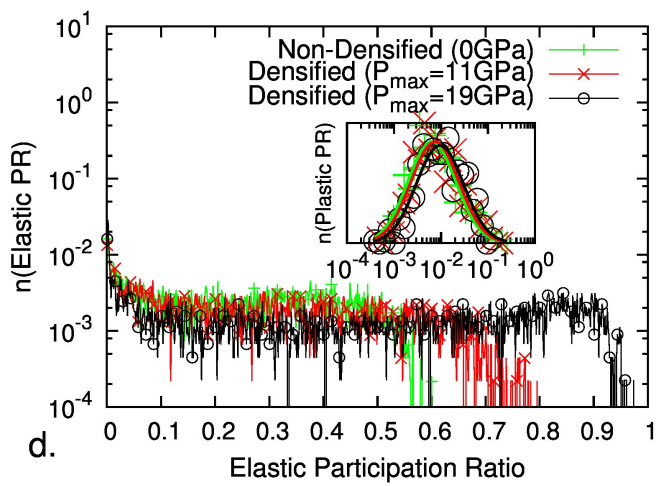

Figure 11: [Color Online] a) Amplitude of the Non-Affine displacement field (departure from homogeneous shear) during an irreversible rearrangement along shear band. (Picture realised with Atomeye [34]) b) Distribution of the amplitudes of the Non-Affine displacement fields measured on each atom for different steps in the stress-strain curve, and for samples initially submitted to different $P_{\max }$. c) Histograms of the second order moments of the Non-Affine displacement field obtained from all the configurations obtained during a shear at constant volume, for samples initially submitted to different $P_{\max }$. Same legend as in b. Note the difference between the elastic and the plastic events. d) Histogram of the participation ratio of the Non-Affine displacement field during the shear deformation at constant volume for the same samples. Main graph : for elastic events. Inset : for plastic events. 

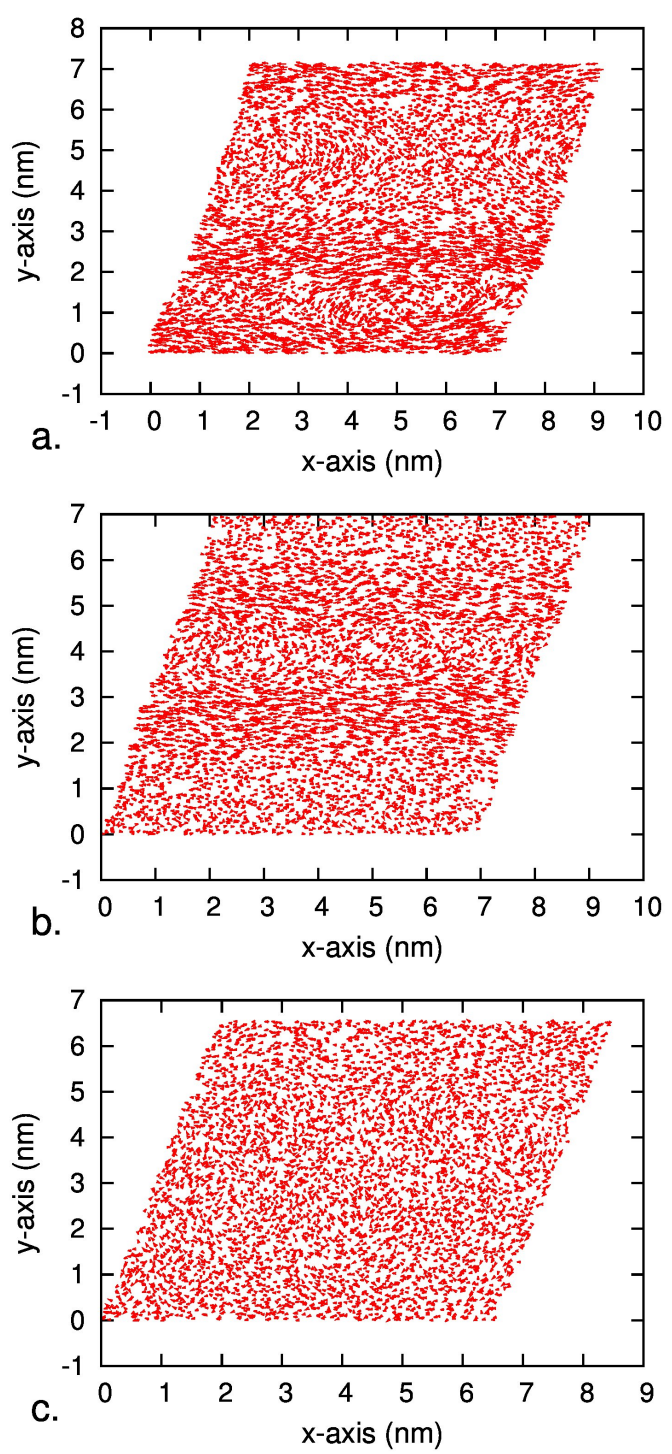

Figure 12: [Color Online] Shear flow from 15 to 30 percents of deformation a) for non-densified sample, b) for sample initially submitted to $P_{\max }=11 G P a$, c) for sample initially submitted to $P_{\max }=19 G P a$. The arrow sizes that shows the atomic displacements have been increased by a factor 10 .

teresting to look at the evolution of the hydrostatic pressure with shear. Fig. 10-a shows that the pressure first decreases, up to the maximum stress, and then increases again when shear softening sets in. After the maximum stress has been reached (that is during the softening and then during the plastic flow), the pressure evolves linearly with shear stress, following a Mohr-Coulomb like relation with a negative coefficient. In the plastic plateau, pressure and shear stress are thus proportional, with the same negative proportionality coefficient. The negative sign of this coefficient is simply related to the fact that a deformation at constant volume hides the natural densification of the system, and constant volume is maintained by tension $\left(\sigma_{x y}>0\right.$ but $P<0$ ). This behaviour is very characteristic of pure silica samples. It is for example opposite to the behaviour of granular materials [25], where shear induces dilatancy $(P>0)$; this negative slope also disappears in silicate glasses $\left(x . \mathrm{SiO}_{2}+(1-x) \cdot \mathrm{Na}_{2} \mathrm{O}\right)$ along with the propensity to densify. It is interesting to note that in our samples, the shear stress always dominates over other stress components except at the very beginning of the shear deformation (Fig. 10-b). Idem for the compressive (or tensile) stress $\sigma_{y y}$ that dominates over the pressure. The Mohr-Coulomb like behaviour could thus be as precisely described by a Drucker-Prager like behaviour (where the maximum deviatoric stress would be replaced by the amplitude of the deviatoric stresses, and the tensile stress by the total pressure). The difference between the two behaviours is not visible in our data. This is why we refer more generally to a "Mohr-Coulomb like" behaviour (that we describe here simply by the linear dependance between shear stress and pressure), and we omit more details of the behaviour law.

In the present case of shear deformation, the corresponding non-affine displacements are very different in the elastic reversible part and in the plastic part. Elastic rearrangement shows homogeneous displacements spread over the whole system (Fig. 11); then quadrupolarlike dissipative rearrangements occur even before the maximum shear stress is reached, in the linear part of the stress-strain curve. Finally, the plastic plateau consists in a series of localized rearrangements and shear bands spanning the system along a plane parallel to the shear direction. This confirms the localization of plastic rearrangements, in contrast to what happens upon pure compression (Part III). The progressive localization of the non-affine displacements can be quantified by computing the participation ratio as in the previous part. We show in Fig. 11-c that the participation ratio in the sheared systems is largely distributed. The elastic rearrangements have a large P.R., with a maximum value depending on the density of the sample $\left(P . R \cdot \max =50 \%\right.$ for $P_{\max }=0 G P a$ and P.R. $\max =95 \%$ for $\left.P_{\max }=19 G P a\right)$, thus confirming the progressive homogeneization of the non-affine displacements with pressure. This progressive homogeneization is also confirmed by the distribution of the variance of the non-affine displacement field (Fig. 11-b). Its minimum value is decreased by a factor of 10 when $P_{\max }$ increases from 0 to $19 G P a$. The displacement's fluctutations in the elastic regime can then have very small values. The Participation ratio of plastic rearrangements are centered on a second peak at smaller values depending on the system size but not on the density. For $N=24000$, this peak is centered on P.R. $=10^{-2}$ corresponding to $1 \%$ of atoms that is 240 atoms, that is approximately a cube delimited by $\approx 6$ atoms on each side. For $N=192000$ the maximum value is decreased to $1.2 .10^{-3}(0.12 \%)$ corresponding to the same number of atoms (not shown 
here). These small values for plastic rearrangements thus correspond to very localized, size independent, rearrangements. They can be compared to the values obtained upon compression. In the latter case, the participation ratio is size independent, with an average about 0.4 as for elastic rearrangements. Note also that the maximum value of the relative amplitude of the Non-Affine displacements (Fig. 11-(b) and Fig. 8-(b)), as compared with the displacement applied at the boundaries, is between $10^{3}$ and $10^{5}$ times larger during shear at constant volume than during a pure compression. The consequence is that the effect of the inhomogeneous strain can be quantitatively far more important during a shear than during a compression.

\section{DISCUSSION AND CONCLUSION}

We have shown in this paper that the present model silica glass exhibits a robust mechanical response. Overall, our results reproduce all the main features of the mechanical behaviour of amorphous silica. In hydrostatic compression the anomalous elastic softening of the Bulk Modulus with pressure is recovered. The loading history affects the structure and the mechanical response only if the system exceeds the yield threshold which is $11 \mathrm{GPa}$ for the pristine sample. The yield threshold is the maximum pressure reached in the single load, after the initial threshold has been exceeded. It increases with the densification of the sample. This corresponds to the strain hardening which has been evidenced experimentally [15]. Cyclic loading however converges to the single load behaviour when the previous maximum pressure is approached from below, thus showing that the system keeps somehow the structural memory of its initially densified state. The cyclic behaviour also refered to as microplasticity [44], is reminiscent of a first order phase transition observed in the dynamics of many other disordered systems [63]. We understand it here by the discordance between Local and Macroscopic Bulk Modulus, and the occurrence of micro-instabilities.

In shear, we obtain reasonable values both for the modulus and the yield threshold. We evidence that the shear stress threshold decreases with pressure as expected. The reason is the suppression of shear softening when the system is homogeneized by irreversible compression.

As already mentioned, the present model emulates the complex mechanical response of silica reasonably well although it is not a quantitative model. In particular we note that the very low value of the Poisson coefficient is not rendered correctly here.

One of the salient features of our results is the presence of small scale jumps both for one shot and for cyclic loadings. These jumps are plastic rearrangements at small scale, which occur between nearby configurations. Similar jumps are also known at larger lengthscales in some bistable cristalline phases [7]. They lead to open loops in reversible compression curves because of the dissipation associated with the jumps due to internal friction. In this paper, we have shown that these dissipative jumps are directly responsible for the anomalous behaviour of the Bulk Modulus with pressure.

It is expected that the jumps are clearly visible here because of 1) the small scale of the sample and the good pressure resolution 2) the nearly zero temperature. At non-zero temperature, thermal activation would contribute to wash out the jumps. In particular, the recovery of the pristine volume after annealing at temperatures significantly below the glass transition temperature could actually be due to the thermal activation of such jumps out of higher energy configuration [38].

Evidence of structural changes at high pressures is shown in the permanent densification and in the evolution of intertetrahedral angles upon compression, in agreement with experiments. In addition, it is shown here that these structural changes affect the mechanical response (stress-strain behaviour) and especially the stress overshoot characteristic of the occurrence of a permanent shear band. The shear stress overshoot tends to disappear at higher densification, when the system has experienced pressures larger than the macroscopic yield threshold. Our study has shown clearly that this new behaviour is directly related to the spatial homogenization of the displacement field. The departure from the homogeneous displacement field is more pronounced at small pressures. The effect of pressure in our model silica glass, is thus to homogeneize the mechanical response : the shear at imposed pressures would give rise probably to smooth stress-strain behaviour and homogeneous displacement fields. We have not been able however to connect the displacement field to the structural changes in our sample. The silica sample has a natural tendency to densify. But the microscopic origin of this behaviour is far from been clearly established.

Note finally that all these results have been obtained on a model silica glass. The use of this model was encouraged by the very promising comparison with experimental results. This study has been performed at very low temperature in order to emphasize the pure geometrical effects on the mechanical response of the sample, and compare it to previously measured data. The addition of thermal noise could affect them strongly, as well as the use of more realistic quenching rates however not reachable presently by Molecular Dynamics Simulations. Further studies on shear at constant pressure and on soda-lime glasses are under way. Indeed the atomic mechanisms evidenced here can be very sensitive to the chemical composition of the glass. As shown experimentally, the mechanical behaviour would probably be very different for example for soda-lime glasses [23, 30] where the presence of cations repealling each other is not favourable to densification. The quantitative comparison with Silicate Glasses will be the subject of a future study [52]. 
[1] F. Albano and M. Falk, The Journal of Chemical Physics 122, 154508 (2005) ; Y.Shi and M.L. Falk, Phys. Rev. B 73, 214201 (2006); Y.Q. Cheng, A.J. Cao, H.W. Sheng and E. Ma, Acta Materialia 56, 5263 (2008).

[2] A.S. Argon, Acta Metallurgica 27, 47 (1979).

[3] M.J. Demkowicz and A.S. Argon, Phys. Rev. Lett. 93, 025505 (2004).

[4] M. J. Demkowicz and A. S. Argon, Phys. Rev. B 72, 245205 (2005).

[5] G. Baldi, V.M. Giordano and G. Monaco, Phys. Rev. B 83, 174203 (2011)

[6] J.C. Baret, D. Vandembroucq and S. Roux, Physical Review Letters 89, 195506 (2002); M. Talamali, V. Petaja, S. Roux and D. Vandembroucq, arXiv :1005.2463 (2010).

[7] M.W. Barsoum et al., Nature Mat. 2107 (2003).

[8] M. Benoit, S. Ispas, P. Jund and R. Julien, Euro. Phys. J. B 13, 631 (2000)

[9] B.W.H. van Beest, G.J. Kramer and R.A. van Santen, Physical Review Letters 64, 1955 (1983)

[10] M. I. Huggins and J. E. Mayer, Jour. of Chem. Phys. 1, 643 (1993).

[11] A. D. Buckingham, P. W. Fowler, J. M. Hutson, Chem. Rev. 68, 963 (1988).

[12] A. Carre, L. Berthier, J. Horbach, S. Ispas and W. Kob, Jour. of Chem. Phys. 127, 114512 (2007)

[13] P.G. Coombs, J.F. De Natale, P.J. Hood, E.K. McElfresh, R.S. Wortman and J.F. Schackelford, Phil. Mag. 51, L39 (1985)

[14] Da Silva et al. Phil. Mag. 31, 713 (1975).

[15] D. Vandembroucq, T. Deschamps, C. Coussa, A. Perriot, E. Barthel, B. Champagnon and C. Martinet, Journal of Physics : Condensed Matter 20, 485221 (2008)

[16] S. N. Taraskin, S. I. Simdyankin, S. R. Elliott, J. R. Neilson, and T. Lo, Phys. Rev. Lett. 97, 055504 (2006)

[17] C. Sonneville, T. Deschamps, C. Martinet, B. Champagnon, to be published 2011.

[18] P.P. Ewald, Ann. Phys. (Leipzig) 64, 253 (1921).

[19] M.L. Falk and J.S. Langer, Physical Review E 57, 7192 (1998).

[20] C. Fusco, T. Albaret and A. Tanguy, Phys. Rev. E 82, 066116 (2010)

[21] F. Lechenault, G. Pallares, M. George, C. Rountree, E. Bouchaud and M. Ciccotti, Physical Review Letters 104, 025502 (2010).

[22] C. Goldenberg, A. Tanguy and J.-L. Barrat, Europhysics Letters 80, 16003 (2007).

[23] T.M. Gross and M. Tomozawa, Journal of Applied Physics 104, 063529 (2008).

[24] R.C. Zeller and R.O. Pohl, Physical Review B 4, 2029 (1971)

[25] F. Lacombe, S. Zapperi and H.J. Herrmann, The European Physical Journal E 2, 10 (1999)

[26] L. Huang and J. Kieffer, J. Chem. Phys. 118, 1487 (2003).

[27] S. Ispas and W. Kob, in preparation (2011)

[28] X. Jia, Physical Review Letters 93, 154303 (2004)

[29] W.L. Johnson and K. Samwer, Physical Review Letters 95, 1995501 (2005).

[30] G. Kermouche, E. Barthel, D. Vandembroucq and Ph. Dubujet, Acta Materialia 56, 3222 (2008)

[31] K. Kondo, S. IiO, and A. Sawaoka, J. Appl. Phys. 52,
2826 (1981) (1981).

[32] J.H. Konnert and J. Karle, Acta Cryst. A 29, 702 (1973)

[33] http ://lammps.sandia.gov

[34] J. Li, Modelling Simul. Mater. Sci. Eng. 11, 173 (2003)

[35] A. Lemaitre and C. Caroli, Physical Review E 76, 036104 (2007).

[36] F. Leonforte, A. Tanguy, R. Boissiere, J.P. Wittmer and J.-L. Barrat, Phys. Rev. B 72, 224206 (2005)

[37] F. Leonforte, A. Tanguy and J.-L. Barrat, Phys. Rev. Lett. 97, $055501(2006)$

[38] J. D. Mackenzie, J. Am. Ceram. Soc. 46, 461 (1963) ; J. D. Mackenzie , J. Am. Ceram. Soc. 46, 470 (1963).

[39] C. Maloney and A. Lemaitre, Phys. Rev. E 74, 016118 (2006).

[40] C.E. Maloney, Physical Review Letters 87, 035503 (2006).

[41] F. Mauri et al. Phys. Rev. B 62, R4786 (2000)

[42] C. Meade, R.J. Hemley and H. K. Mao Phys. Rev. Lett. $691387 ; 96$ ¿1390 (1992).

[43] M. Li, J. Eckert, L. Kecskes and J. Lewandowski, J. Mater. Res. 22, 255 (2007).

[44] Microplasticity, C. J. McMahon Jr. editor, Interscience Publishers, (New-York,1968)

[45] A. Monaco, A.I. Chumakov, G. Monaco, W.A. Crichton, A. Meyer, L. Comez, D. Fioretto, J. Korecki and R. Ruffer, Physical Review Letters 97, 135501 (2006)

[46] P. M. Morse, Phys. Rev. 34, 57 (1929).

[47] R.L. Mozzi and B.E. Warren, J. Appl. Cryst. 2, 164 (1969)

[48] J. Neuefeind and K.D. Liss, arXiv :chem-ph/9603004 (1996)

[49] K. Niss, B. Begen, B. Frick, J. Ollivier, A. Beraud, A. Sokolov, V. N. Novikov, and C. Alba-Simionesco, Physical Review Letters 99, 055502 (2007)

[50] L. Orsingher, A. Fontana, E. Gilioli, G. Carini, Jr., G. Carini, G. Tripodo, T. Unruh and U. Buchenau, The Journal of Chemical Physics 132, 124508 (2010).

[51] M. Parinello and A. Rahman, Journal of Applied Physics 52, 7182-7190 (1981)

[52] M. Pedard, B. Mantisi and A. Tanguy, in preparation (2011)

[53] Pedone et al. J. Phys. Chem. B 110, 11780 (2006)

[54] A. Perriot, V. Martinez, L. Grosvalet, C. Martinet, B. Champagnon, D. Vandembroucq and E. Barthel, J. Am. Ceram. Soc. 89, 596 (2006).

[55] A. Perriot, E. Barthel, G. Kermouche, G. Quérel and D. Vandembroucq, Philosophical Magazine, p.1-10 (2009).

[56] R.F. Pettifer, R. Dupree, I. Farman and U. Sternberg, J. Non-Cryst. Solids 106, 408 (1988)

[57] B.L. Phillips, Phys. Chem. Miner. 20, 341 (1993).

[58] A. Polian and M. Grimsditch, Phys. Rev. B, 416086 (1990).

[59] D. Rodney, A. Tanguy and D. Vandembroucq, http ://arxiv.org/abs/1107.2022

[60] J. Rottler and M. O. Robbins, Phys. Rev. E. 68, 011507 (2003); J. Rottler and M. O. Robbins, Comp. Phys. Comm. 169, 177 (2005).

[61] B. Ruffle, G. Guimbretiere, E. Courtens and R. Vacher, Physical Review Letters 96, 045502 (2006)

[62] W. Schirmacher, C. Tomaras, B. Schmid, G. Baldi, G. Viliani, G. Ruocco and T. Scopigno, Condensed Matter 
Physics 13, 23605 (2010); C. Tomaras, B. Schmid and W. Schirmacher, Physical Review B 81, 104206 (2010).

[63] J.P. Sethna, K. Dahmen, S. Kartha, J.A. Krumhansl, B.W. Roberts and J.D. Shore, Physical Review Letters 70, 3347 (1993).

[64] P. Sollich, Phys. Rev. E 58, 738 (1998).

[65] F. Spaepen, Acta Metallurgica 25, 407 (1977).

[66] M. Talati, T. Albaret and A. Tanguy, Europhys. Lett. 86, 66005 (2009).

[67] A. Tanguy, J.P. Wittmer, F. Leonforte and J.-L. Barrat, Phys. Rev. B 66, 174205 (2002).

[68] A. Tanguy, F. Leonforte and J.-L. Barrat, Eur. Phys. J. E 20, 255 (2006).

[69] A. Tanguy, B. Mantisi and M. Tsamados, Europhysics Letters 90, 16004 (2010).

[70] S.N. Taraskin and S.R. Elliott, Phys. Rev. B 56, 8605 (1997).

[71] K. Trachenko, M.T. Dove, V. Brazhkin and F.S. El'kin, Physical Review Letters 93135502 (2004).

[72] M. Tsamados, A. Tanguy, C. Goldenberg and J.-L. Barrat, Phys. Rev. E 80, 026112 (2009)
[73] M.. Tsamados, European Physical Journal E 32, 165 (2010).

[74] F. Varnik, L. Bocquet, J.-L. Barrat and L. Berthier, Physical Review Letters 90, 095702 (2003).

[75] F. Varnik, L. Bocquet and J.-L. Barrat, J. Chem. Phys. 120, 2788 (2004).

[76] Vashita et al. Phys. Rev. B 41, 17 (1990).

[77] K. Vollmayr, W. Kob and K. Binder, Phys. Rev. B 54, 15808 (1996).

[78] J.R. Willis, Adv. Appl. Mech. 21, 2 (1981).

[79] D. Wolf, P. Keblinski, S. R. Phillpot, and J. Eggebrecht, J Chem. Phys. 110, 17 (1999).

[80] D.I. Grimley, A.C. Wright and R.N. Sinclair, J. NonCryst. Solids 119, 49 (1990) ; A.C. Wright, J. Non-Cryst. Solids 179, 84 (1994).

[81] P. Xhonneux, E. Courtens, J. Pelous, and R. Vacher, Europhys. Lett. 10, 733 (1989)

[82] M. Yukawa, Proc. Phys. Math. Soc. Jpn. 17, 48 (1935).

[83] C.-S. Zha, R. J. Hemley, H.-K. Mao, T. S. Duffy and C. Meade, Phys. Rev. B 50, 13105 (1994) 